\title{
DISCLAIMER
}

This report was prepared as an account of work sponsored by an agency of the United States Government. Neither the United States Government nor any agency thereof, nor any of their employees, makes any warranty, express or implied, or assumes any legal liability or responsibility for the accuracy, completeness, or usefulness of any information, apparatus, product, or process disclosed, or represents that its use would not infringe privately owned rights. Reference herein to any specific commercial product, process, or service by trade name, trademark, manufacturer, or otherwise does not necessarily constitute or imply its endorsement, recommendation, or favoring by the United States Government or any agency thereof. The views and opinions of authors expressed herein do not necessarily state or reflect those of the United States Government or any agency thereof.

MAD-PH-728

OTIS-499

November 1992

\section{A Separate Higgs?}

V. BARGER ${ }^{a}$, N.G. DESHPANDE ${ }^{b}$, J.L. HEWETT ${ }^{b, c}$, and T.G. RIZZO ${ }^{b, c}$

a Department of Physics, University of Wisconsin, Madison, WI 53706

${ }^{b}$ Institute of Theoretical Science, University oí Oregon, Eugene, OR 97403

${ }^{c}$ High Energy Physics Division, Argonne National Laboratory, Argonne, IL 60439

\begin{abstract}
We investigate the possibility of a multi-Higgs doublet model where the lightest neutral Higgs boson $\left(h^{0}\right)$ decouples from the fermion sector. We are partially motivated by the four $\ell^{+} \ell^{-} \gamma \gamma$ events with $M_{\gamma \gamma} \simeq 60 \mathrm{GeV}$ recently observed by the L3 collaboration, which could be a signal for $Z \rightarrow\left(Z^{*} \rightarrow \ell^{+} \ell^{-}\right)+\left(h^{0} \rightarrow \gamma \gamma\right)$. Collider signatures for the additional physical Higgs bosons present in such models are discussed.
\end{abstract}


Although the predictions of the Standard Model (SM) are in excellent agreement with data[1], the symmetry breaking mechanism responsible for generating fermion and gauge boson masses remains mysterious. In the SM, a single scalar doublet is the source of spontaneous symmetry breaking via the Higgs mechanism[2]. However, the possibility of an enlarged Higgs sector beyond the minimal one-doublet version is consistent with data, and is naturally present in many theories which go beyond the SM. It is also possible that the symmetry breaking mechanism responsible for giving masses to gauge bosons is separate from that which generates the fermion masses [3]. An example is technicolor models, where fermion masses arise from extended technicolor. Since experimental searches for the remnants of spontaneous symmetry breaking depend sensitively on the nature of the scalar sector, all model possibilities must be explored. In this context, we examine a scenario wherein the lightest CP-even neutral Higgs field $\left(h^{0}\right)$ does not couple to fermions, but has essentially SM-like couplings to the $W$ and $Z$ gauge bosons. If such a particle were sufficiently light, it would be produced at LEP via the reaction $e^{+} e^{-} \rightarrow Z^{*} h^{0}$ and an excess of $\ell^{+} \ell^{-} \gamma \gamma$ or $q \bar{q} \gamma \gamma$ final states could be observed since then the $2 \gamma$ decay of $h^{0}$ would be dominant. The L3 Collaboration has recently reported four $\ell^{+} \ell^{-} \gamma \gamma$ events with $M_{\gamma \gamma} \simeq 60 \mathrm{GeV}[4]$ which are not explained by SM processes. Motivated by the L3 result, we explore the simplest scenario in which the $h^{0}$ properties above are obtained.

A simple realization is possible in a model with two Higgs doublets, one of which primarily generates the $W$ and $Z$ boson masses and the other is coupled to the fermions. This is often referred to as model I in the literature[2]. Denoting the multiplets by $\phi_{1,2}$, we impose the discrete symmetry $\phi_{1} \leftrightarrow-\phi_{1}$ under which the full Lagrangian and the fermion fields are fully invariant. This ensures that only $\phi_{2}$ couples to fermions and generates the fermion masses and also guarantees that tree-level flavor changing neutral currents are absent. Demanding CP invariance for simplicity, the most general Higgs potential is then 
given by[2]

$$
\begin{gathered}
V=\lambda_{1}\left(\phi_{1}^{\dagger} \phi_{1}-v_{1}^{2}\right)^{2}+\lambda_{2}\left(\phi_{2}^{\dagger} \phi_{2}-v_{2}^{2}\right)^{2}+\lambda_{3}\left[\left(\phi_{1}^{\dagger} \phi_{1}-v_{1}^{2}\right)+\left(\phi_{2}^{\dagger} \phi_{2}-v_{2}^{2}\right)\right]^{2} \\
+\lambda_{4}\left[\left(\phi_{1}^{\dagger} \phi_{1}\right)\left(\phi_{2}^{\dagger} \phi_{2}\right)-\left(\phi_{i}^{\dagger} \phi_{2}\right)\left(\phi_{2}^{\dagger} \phi_{1}\right)\right]+\lambda_{6}\left[\operatorname{Im}\left(\phi_{1}^{\dagger} \phi_{2}\right)\right]^{2}
\end{gathered}
$$

Here the Yukawa couplings $\lambda_{i}$ are real, and $v_{1,2}$ are the vacuum expectation values (VEV) of $\phi_{1,2}$ which are subject to the constraint $v_{1}^{2}+v_{2}^{2} \equiv v^{2}$, where $v$ is the usual VEV of the SM. The ratio of VEV's is parameterized as $\tan \beta \equiv v_{2} / v_{1}$. If we had tried to construct a model wherein CP was spontaneously violated and the symmetry $\phi_{1} \leftrightarrow-\phi_{1}$ was strictly enforced we would find that these two demands are incompatible[5]. As usual, the Higgs mass spectrum will consist of a neutral CP-odd field, $A\left(m_{A}=v \sqrt{\lambda_{6}}\right)$, a pair of charged scalars, $H^{ \pm}\left(m_{H^{ \pm}}=v \sqrt{\lambda_{4}}\right)$, and a pair of CP-even scalars whose mass matrix is given by Eq. (4.15) in Ref. 2 with $\lambda_{5}$ now set to zero. Diagonalization of this matrix through a rotation by an angle $\alpha(-\pi / 2 \leq \alpha \leq 0)$ gives the mass eigenstate basis $\left\{h^{0}, H^{0}\right\}$ with $m_{h^{0}}<m_{H^{0}}$ by definition. In terms of $\alpha$ and $\beta$, the couplings of $h^{0}, H^{0}, A$, and $H^{ \pm}$to quarks and leptons are given in Ref. 2. It is the field $h^{0}$ that we will decouple from fermions while maintaining its couplings to the $W$ and $Z$ at close to SM strength.

In order to sufficiently inhibit the $h^{0} f \bar{f}$ couplings, it is immediately apparent from the structure of the couplings that we must restrict $\cos \alpha / \sin \beta \ll 1$. In practice, we will require $\alpha$ to be within $\simeq 10^{-3}$ of $-\pi / 2$, which implies that $\lambda_{3} v_{1} v_{2} \ll \lambda_{i} v_{i}^{2}(i=1,2)$ and results in $m_{h^{0}} \simeq 2 \sqrt{\lambda_{1}} v \cos \beta$ and $m_{H^{0}} \simeq 2 \sqrt{\lambda_{2}} v \sin \beta$ as well as $\lambda_{2}>\lambda_{1} / \tan ^{2} \beta$. There is no symmetry that will allow us to set $\lambda_{3}=0$, and therefore the parameters in the Higgs potential must be fine-tuned to achieve sufficient decoupling. Even if we tune $\alpha$ to $-\pi / 2$ at the tree-level and force the $h^{0} f \vec{f}$ coupling to zero, loop diagrams involving both $W^{ \pm}$and $H^{ \pm}$contributions will induce such couplings proportional to $m_{f}$. As in other fine tuned scenarios, we must choose the value of $\alpha$ at the one-loop level to insure that the $h^{0} \rightarrow f \bar{f}$ rates remain small. 
Since $h^{0} \rightarrow f \bar{f}$ is forbidden, the dominant decay of $h^{0}$ when the $h^{0}$ lies below the $W W^{*}$ threshold is to the $\gamma \gamma$ final state through the induced $h^{0} \gamma \gamma$ coupling at one-loop. In order to calculate the rate for $h^{0} \rightarrow \gamma \gamma$ (and for $H^{0}, A \rightarrow \gamma \gamma$ ), we need the $h^{0} H^{+} H^{-}$, $\mathrm{H}^{0} \mathrm{H}^{+} \mathrm{H}^{-}$, and $\mathrm{AH}^{+} \mathrm{H}^{-}$couplings. In this model we obtain

$$
\begin{aligned}
g_{h^{0} H^{+} H^{-}} & =\frac{-m_{h^{0}}^{2} \sin ^{2} \beta}{\sqrt{2} v \cos \beta}-\frac{m_{H^{ \pm}}^{2} \sqrt{2} \cos \beta}{v} \\
g_{H^{0} H^{+} H^{-}} & =\frac{m_{h^{0}}^{2} \cos ^{2} \beta}{\sqrt{2} v \sin \beta}+\frac{m_{H^{ \pm}}^{2} \sqrt{2} \sin \beta}{v} \\
g_{A H^{+} H^{-}} & =0
\end{aligned}
$$

where for numerical purposes we have set $\lambda_{3}=0$ and $\alpha=-\pi / 2$. Since the $A H^{+} H^{-}$coupling vanishes, the $A \rightarrow \gamma \gamma$ process can proceed only via fermion loops. For $m_{h^{0}}=60 \mathrm{GeV}$, we find $\Gamma\left(h^{0} \rightarrow \gamma \gamma\right)=1.98 \mathrm{MeV}$.

For substantial production of $h^{0}$ in association with a $Z^{*}$ at LEP via $e^{+} e^{-} \rightarrow Z^{*} h^{0}$, the $Z Z^{*} h^{0}$ coupling $(\sim \sin (\beta-\alpha))$. must be as large as possible. With $\alpha$ very close to $-\pi / 2$, we note that $\sin (\beta-\alpha) \simeq 1-\frac{1}{2} \beta^{2}+\mathcal{O}\left(\beta^{4}\right)$ if $\beta \ll 1$, and thus we will require $\beta$ to be small. For $\sin (\beta-\alpha) \simeq 1$, the branching fraction for $Z \rightarrow Z^{*} h^{0} \rightarrow e^{+} e^{-} h^{0}$ is $0.36 \times 10^{-6}$ for $m_{h^{0}}=60 \mathrm{GeV}$. [L3 has a total of $\sim 10^{6} Z$ events.] However, $\beta$ cannot be too small if the couplings of $H^{ \pm}, H^{0}$, and $A$ are to remain perturbative and be consistent with low-energy data. For $m_{t}=150 \mathrm{GeV}$, such arguments[7] indicate that $\tan \beta \gtrsim 1 / 4$. Henceforth we restrict $\beta$ to lie in the relatively narrow region $1 / 5 \lessgtr \beta \lesssim 1 / 2$ to give SMlike coupling strength to $Z Z^{*} h^{0}$ and still satisfy the perturbative constraints. For $\beta$ in this range and $m_{t}=150 \mathrm{GeV}$, the current experimental bound[8] on the decay $b \rightarrow s \gamma$ places a reasonably strong constraint[7,9] on $m_{H^{ \pm}}$. We obtain $m_{H^{ \pm}} \gtrsim 45(250,420) \mathrm{GeV}$ for $\tan \beta=1 / 2(1 / 3,1 / 4)$, which suggests that $H^{ \pm}$may be quite heavy and then $t \rightarrow H^{+} b$ decay would not occur. 
To ascertain that the $h^{0} \rightarrow \gamma \gamma$ mode dominates $h^{0}$ decay, we must also examine the rates for such modes as $h^{0} \rightarrow W^{*} W^{*}, Z^{*} Z^{*}$ which have so far only been calculated within the SM context[6]. With $\alpha=-\pi / 2, m_{h^{0}}=60 \mathrm{GeV}$, and $\tan \beta$ in the range above we find that the partial widths for these two process is smaller than that for the $\gamma \gamma$ mode by factors of order $20-100$.

If $Z \rightarrow Z^{*} h^{0} \rightarrow \ell^{+} \ell^{-} \gamma \gamma$ is the source of the $\mathrm{L} 3$ events, other $Z^{*}$ decay modes should also be observed. L3 has searched[10] for the process $e^{+} e^{-} \rightarrow Z^{*} h^{0} \rightarrow q \bar{q} \gamma \gamma$ and has placed a bound on the cross section of $\simeq 1 \mathrm{pb}$ for $m_{h^{0}} \simeq 50-60 \mathrm{GeV}$. Using the ratio of branching fractions $B(Z \rightarrow q \bar{q}) / B\left(Z \rightarrow e^{+} e^{-}\right)=20.85$ measured at LEP[1], this bound can be translated into a constraint on the branching fraction for $Z \rightarrow Z^{*} h^{0} \rightarrow e^{+} e^{-} \gamma \gamma$. The result is $B \lessgtr 0.84 \times 10^{-6}$, which is not far above the model prediction of $B=0.36 \times 10^{-6}$. Thus a factor of $\sim 2$ increase in statistics should also reveal the $h^{0}$ signal in the $q \bar{q} \gamma \gamma$ mode. Additionally, since $B(Z \rightarrow \nu \bar{\nu}) / B\left(Z \rightarrow e^{+} e^{-}\right) \simeq 6$, the LEP experiments should also observe approximately 3 times as many $2 \gamma$ plus missing energy events as the sum of $e^{+} e^{-} \gamma \gamma$ and $\mu^{+} \mu^{-} \gamma \gamma$ events.

Since the $W^{\mp} H^{ \pm} H^{0}, Z H^{0} A, h^{0} W^{+} W^{-}$, and $h^{0} Z Z$ couplings all scale as $\sin (\beta-\alpha)$, they will all be large; the $W^{\mp} H^{ \pm} h^{0}, Z h^{0} A, H^{0} W^{+} W^{0}$, and $H^{0} Z Z$ couplings, which scale as $\cos (\beta-\alpha)$, will all be of $\mathcal{O}(\beta)$ and will be suppressed. This has important implications for the production of the heavy $H^{0}$ at the SSC or LHC. (i) The process $g g \rightarrow H^{0} \rightarrow W^{+} W^{-}$will be modified in two ways relative to that of the SM. First, since the $g g H^{0}$ coupling is induced by fermion loops, it will be enhanced by a factor of $\sin \alpha / \sin \beta$. Second, the $H^{0} W^{+} W^{-}$ coupling is suppressed by a factor of $\cos (\beta-\alpha)$. With $\alpha \simeq-\pi / 2$ and $1 / 5 \lessgtr \beta \lesssim 1 / 2$, the resulting production cross section is essentially that of the SM Higgs boson. (ii) The rate for $W^{+} W^{-} \rightarrow H^{0} \rightarrow W^{+} W^{-}$is suppressed by a factor of $\cos ^{4}(\beta-\alpha) \simeq \beta^{4}$, making this process unimportant for the production of a heavy $H^{0}$. (iii) For $H^{0}$ 's with intermediate mass, the $W W^{*}$ decay mode is suppressed in comparison to the SM, but the $H^{0} \rightarrow \gamma \gamma$ 
decay is enhanced. Hence the number of $H^{0} \rightarrow \gamma \gamma$ events for $H^{0}$ in this mass range will be larger than in the SM case. For example, $R_{H}=\sigma\left(g g \rightarrow H^{0} \rightarrow \gamma \gamma\right) / \sigma\left(g g \rightarrow H_{S M}^{0} \rightarrow\right.$ $\gamma \gamma)=57(19,18)$ for $m_{H^{0}}=90(120,150) \mathrm{GeV}$. A similar, but somewhat smaller result, $R_{A}$, is obtained for the corresponding $g g \rightarrow A \rightarrow \gamma \gamma$ process.

Figure 1 shows the ratios $R_{H, A}$ as functions of the respective particle masses; note that $R_{A}$ is always less than $\simeq 1$ for light $A$ but grows rapidly for $m_{A}$ above $140 \mathrm{GeV}$. (iv) The rate for the process $g g \rightarrow H \rightarrow t \bar{t}$ is found to be enhanced in comparison to the SM by a factor of $\sin ^{-4} \beta \simeq 100$ which may render it observable at the SSC/LHC.

Figures $2 \mathrm{a}$ and $2 \mathrm{~b}$ show the $H^{0}$ and $A$ branching fractions, respectively, for $m_{t}=$ $150 \mathrm{GeV}, \tan \beta=1 / 3, \alpha=-\pi / 2$, and $m_{H^{ \pm}}=600 \mathrm{GeV}$. For this choice of parameters, the full widths of both $H^{0}$ and $A$ are comparable to that of the SM Higgs boson. Figure 2 c compares these full widths over a wide range of masses. For a sufficiently massive $H^{0}$ or $A$, the decay into the other physical Higgs fields (i.e., $H^{0} \rightarrow H^{+} H^{-}, 2 A$, etc.) will be dominant. Note that couplings such as $W^{\mp} H^{\ddagger} A$ and $Z H^{+} H^{-}$are fixed by gauge invariance and are unaffected by our choice of the parameters $\alpha$ and $\beta$.

In summary, a two-Higgs doublet model in which one doublet primarily generates the $W, Z$ masses and the other doublet is dominately coupled to fermions has interesting phenomenological implications which deserve further attention. Such a model could account for the $e^{+} e^{-} \gamma \gamma$ and $\mu^{+} \mu^{-} \gamma \gamma$ events observed by the L3 Collaboration. If this interpretation is correct, $\gamma \gamma+$ missing energy and $\gamma \gamma+$ two-jet events should also be found at LEP experiments.

\section{ACKNOWLEDGEMENTS}

The authors would like to thank R.J.N. Phillips for discussions related to this work. J.L.H. and T.G.R. wish to thank the Institute of Theoretical Science at the University of Oregon for their hospitality during the course of this work. This work was supported 
in part by the U.S. Department of Energy under contracts W-31-109-ENG-38, DE-FG85ER400224, and DE-AC02-76ER00881, and in part Texas National Laboratory Commission under grant no. RGFY9173, and in part by the University of Wisconsin Research Committee with funds granted by the Wisconsin Alumni Research Foundation. 


\section{References}

[1] L. Rolandi, ALEPH Collaboration,talk given at the $X X V$ International Conference on High Energy Physics, Dallas, TX, August 1992.

[2] J.F. Gunion, H.E. Haber, G.L. Kane, and S. Dawson, The Higgs Hunters Guide (Addison-Wesley, Redwood City, CA, 1990). For an early discussion of this model, see H.E. Haber, G.L. Kane, and T. Sterling, Nucl. Phys. B161, 493 (1979).

[3] M.S. Berger amd M.S. Chanowitz, Phys. Rev. Lett. 68, 757 (1992); J-L. Basdevant, et al., Argonne National Laboratory report ANL-HEP-PR-92-93 (1992).

[4] L3 Collaboration, CERN Report CERN-PPE/92-152 (1992).

[5] A. Pomarol, Phys. Lett. B287, 331 (1992.)

[6] V. Barger, G. Bhattacharya, T. Han, and B.A. Kniehl, Phys. Rev. D43, 779 (1191).

[7] V. Barger, J.L. Hewett, and R.J.N. Phillips, Phys. Rev. D41, 3421.(1990); G. Buchalla, et al., Nucl. Phys. B355, 305 (1991); J.F. Gunion and B. Grzadkowski, Phys. Lett. B243, 301 (1990.)

[8] D. Kreinick, CLEO Collaboration, talk given at the Beyond the Standard Model III Conference, Ottawa, Canada, June 1992.

[9] J.L. Hewett, talk presented at the 1992 Meeting of the Division of Particles and Fields, Fermilab, Batavia, IL, November 1992.

[10] L3 Collaboration, CERN Report CERN-PPE/92-131 (1992). 


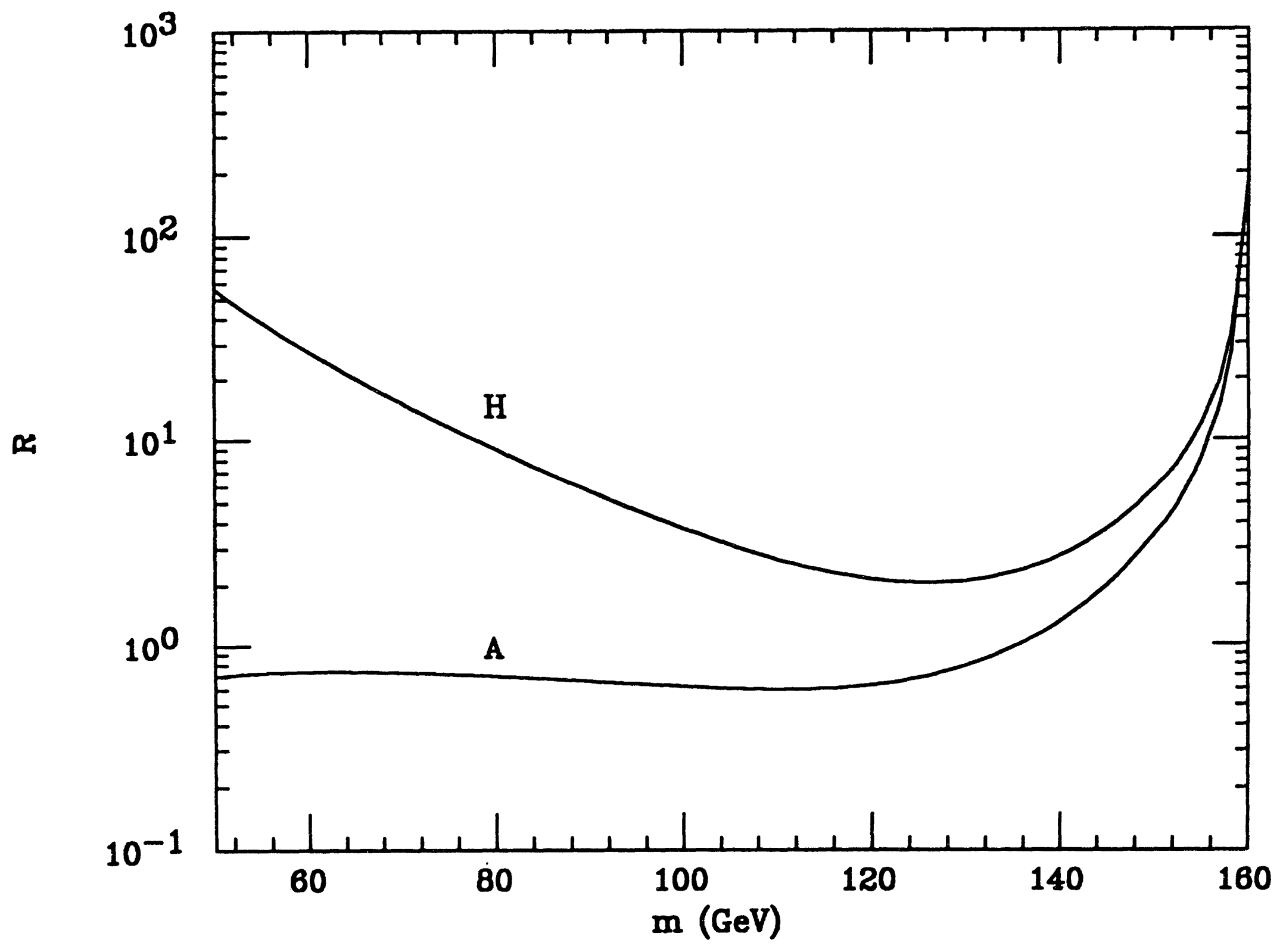

Figure 1 


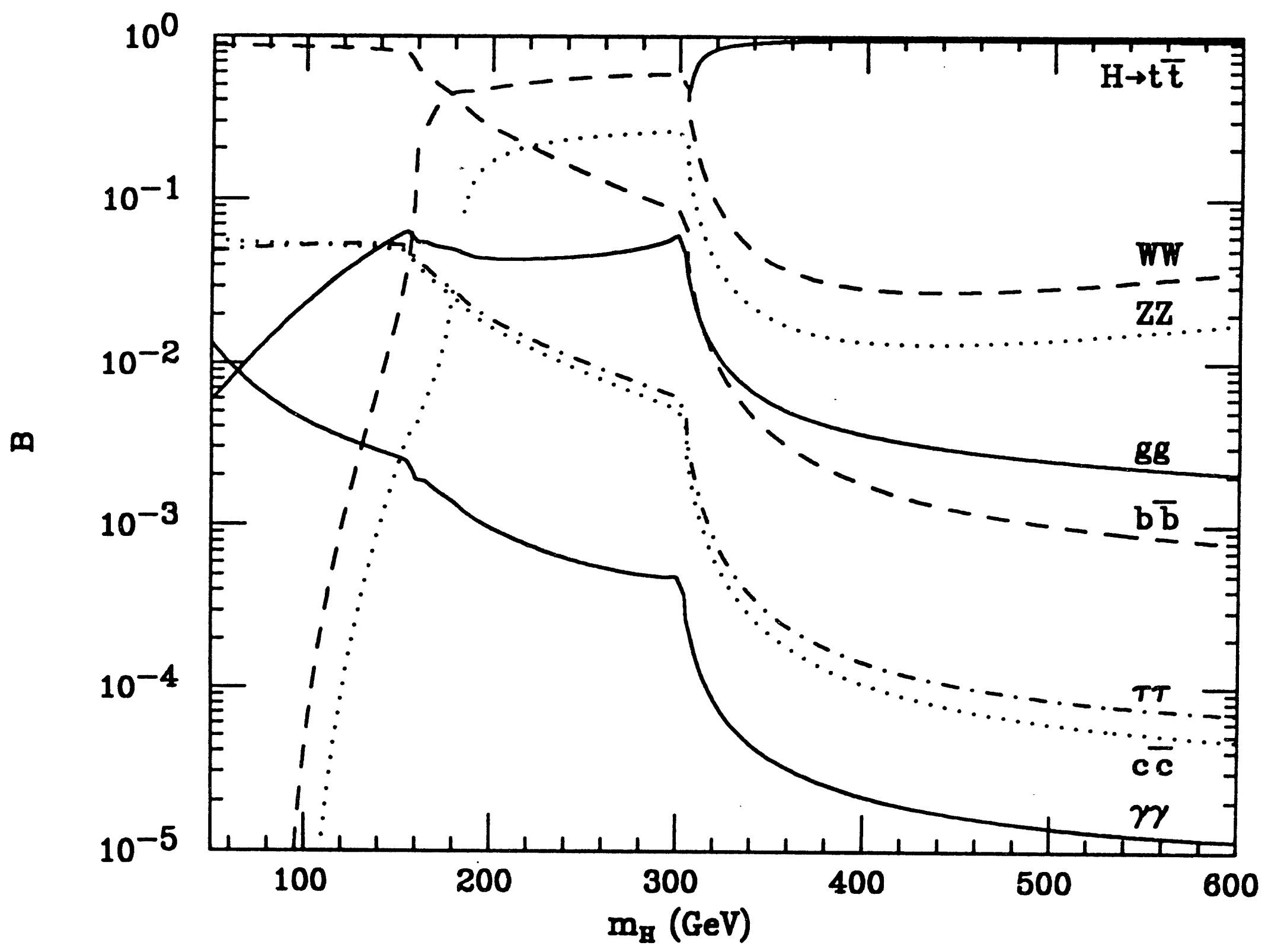

F1gure 2a 


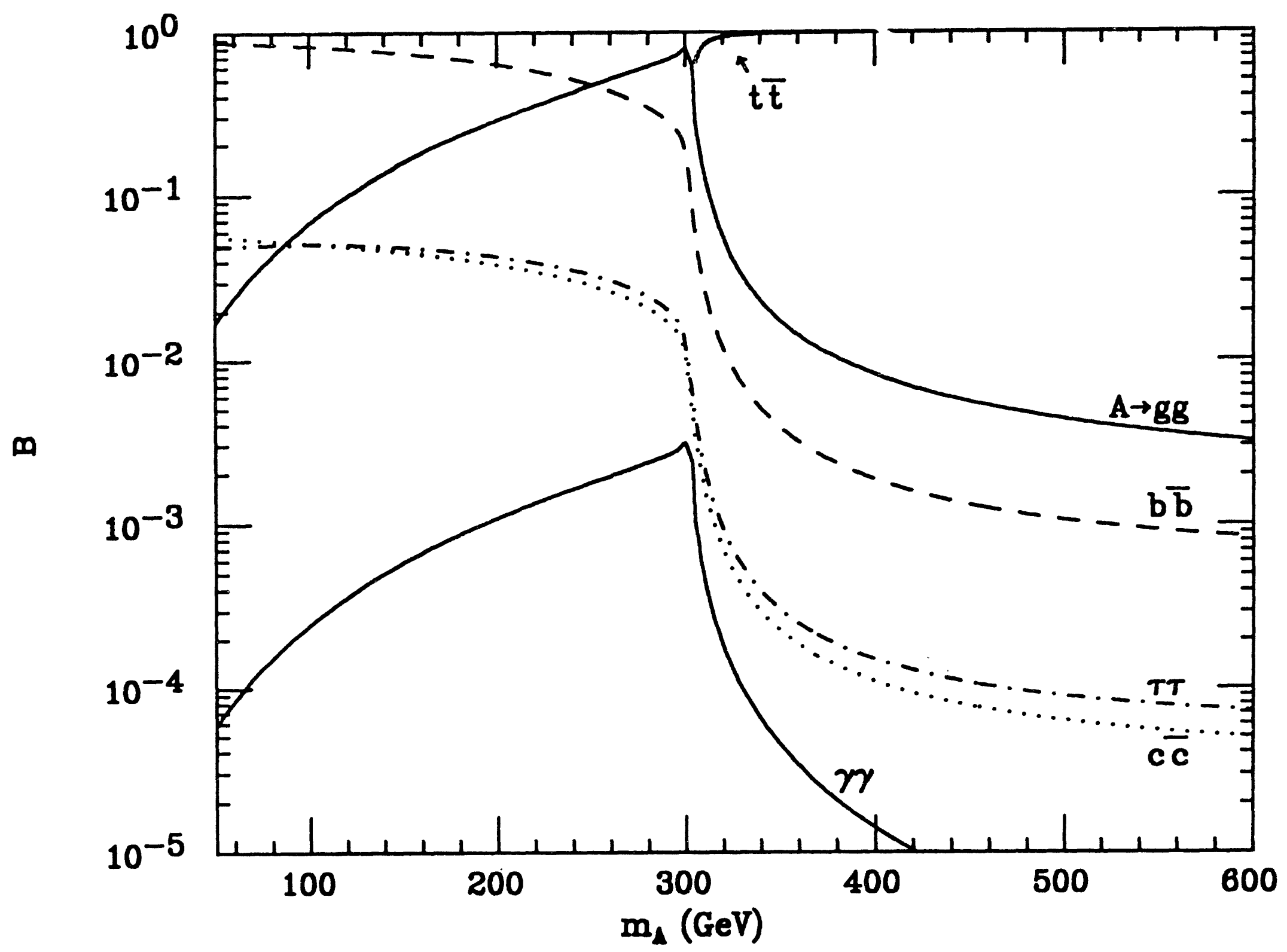




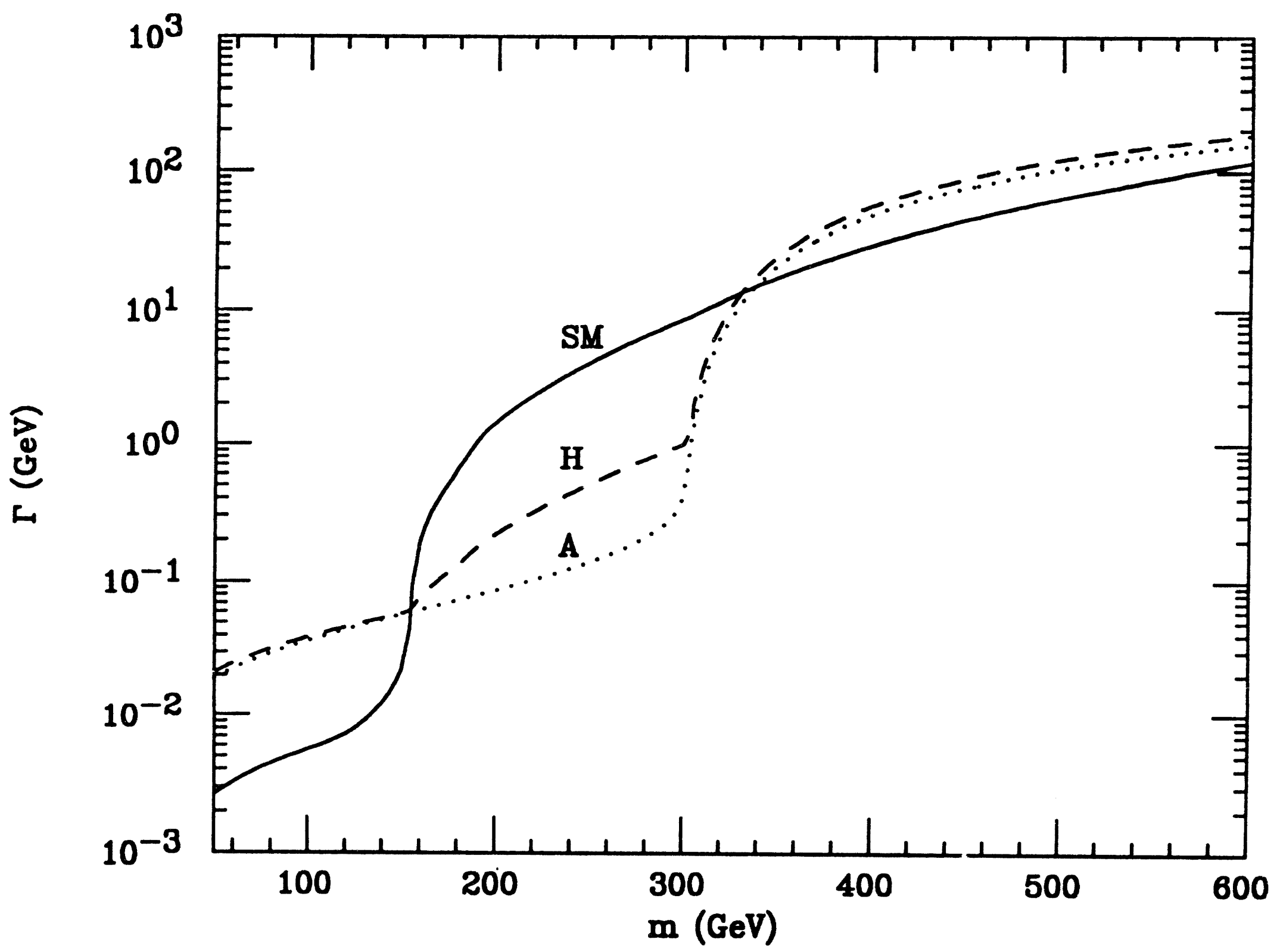

Figure 2c 


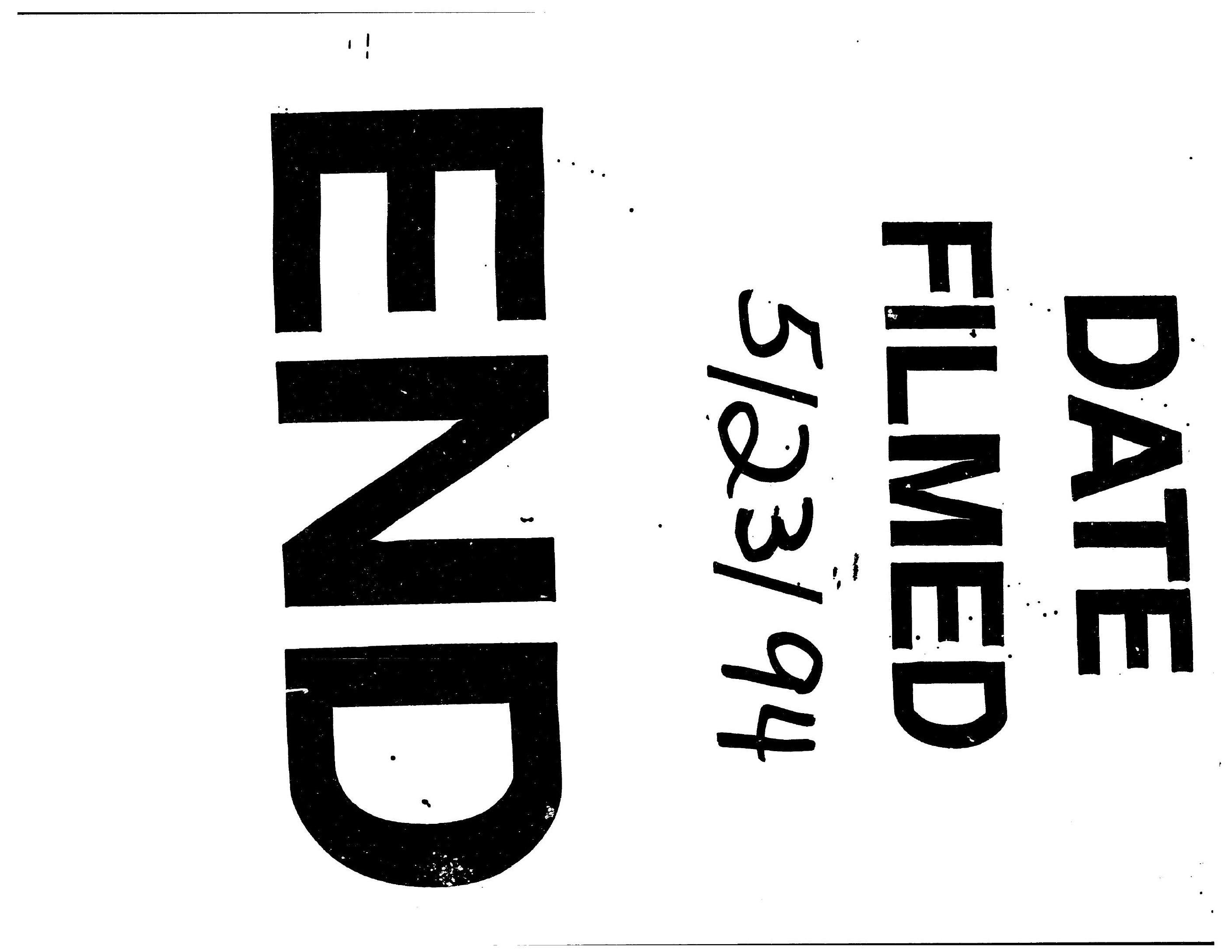


